\title{
Auxiliary differential equation (ADE) implementation of complex-frequency shifted perfectly matched layer (CFS-PML)
}

\author{
Jinzu Ji,Peilin Huang*,Yunpeng Ma \\ ( ${ }^{\star}$ Corresponding author, huang_pei_lin@126.com) \\ School of Aeronautic Science and Engineering, Beihang University, Beijing, 100191, China
}

Keywords: Finite-difference time-domain (FDTD), Perfectly matched layer (PML), Auxiliary differential equation (ADE).

\begin{abstract}
In this paper, an efficient auxiliary differential equation (ADE) method is proposed to implement stretched-coordinate perfectly matched layer (SC-PML) in finite-difference time-domain (FDTD). The derivation of discrete difference operators for the ADE-PML equation for FDTD is also presented. The numerical results show that the ADE-PML's absorbing effect is much better than Mur's second order ABC and RC-PML.
\end{abstract}

\section{Introduction}

The finite-difference time-domain (FDTD) is a convenient tool for solving scattering problems. The main advantages of such a method are that it can be easily applied to conducting obstacles and to dielectric and magnetic obstacles which can be either homogeneous or inhomogeneous [1, 2]. The difficulty encountered when trying to solve open problems, i.e., the domain in which the field has to be computed is unbounded. Since no computer can store an unlimited amount of data, a method has to be used for limiting the domain in which the field is computed. Mur proposed algorithm of one-way approximation of the wave equation and once it is the most used method [3]. Since Berenger proposed perfectly matched layer (PML) method by splitting electric and magnetic field in 1994, The PML absorbing boundary condition (ABC) started a new era for truncating of open calculation region in FDTD [4-7]. The significant advantage of the PML absorbing media is that it provides a mesh truncation that is independent of frequency, wave polarization and incident angle. Moreover, it has extremely small reflection errors. It has been shown that some PML form is material independent, that is, it can truncate any kind of media such as conductor, dielectric and dispersive media [8].

Generally, there are three categories of PMLs: Berenger's split PML [4], Chew's stretched-coordinate PML (SC-PML) [4] and Sacks and Gedney's anisotropic PML (APML) [6, 7]. Berenger's PML splits the electric and magnetic field into two parts and the form is different from Maxwell's equation [4]. APML uses anisotropic media to fulfill the perfectly absorbing feature, but the media's form is very complex which raised much more programming work in application [6, 7]. In truncating different type of media, the former two methods' forms must be modified. SC-PML, which has different form with Maxwell's equation, uses the coordinate transform to implement the PML effect [5]. All the three types of PML is equivalent in mathematics [7]. Kuzuoglu proposed the complex-frequency shifted PML (CFS-PML) to improve the PML's performance [9]. In 2000, Roden proposed convolution PML (CPML) which can truncate any kind of media, since the CPML only modified the curl of the electric and magnetic field [8]. In 2010, Gedney used auxiliary differential equation to implicate CFS-PML [10].

CFS-PML becomes the most accurate and robust choice for truncation of calculation domain. Discrete recursive-convolution (RC) and ADE is the two implementation approaches [8, 10]. The discrete RC method is second-order accurate and is efficient computational cost. With the proper choice of constitutive parameters, this approach provides excellent absorption of both propagation and evanescent waves, and the PML boundary can be placed extremely close to the target under test. The method can also be applied to terminate domains with arbitrary media without any specialization of the implementation. The PML layer's constitutive parameter is dispersive, that is, it 
is frequency dependent. In manipulating dispersive parameter, there are three categories: RC, ADE and ZT (Z-transform) [11]. In PML layer's manipulation, ZT is seldom used.

In this paper, a more efficient form of the CFS-PML expressed via an auxiliary differential equation (ADE) form is presented. The ADE-PML form can be easily extended to multiple-pole PML form. The ADE-PML's performance is validated via numerical experiment.

In this paper, the time dependent factor is $e^{j \omega t}$ and suppressed.

\section{CFS PML in ADE form}

Consider the time-harmonic Maxwell's equation in a lossless, source free media. In the PML region, the equation can be expressed in complex stretched coordinates. For example, the ${ }^{x}$-projection of Faraday's and Ampere's laws are expressed as [5]

$$
\begin{gathered}
-j \omega \mu H_{x}=\frac{1}{S_{y}} \frac{\partial}{\partial y} E_{z}-\frac{1}{S_{z}} \frac{\partial}{\partial z} E_{y} \\
j \omega \varepsilon E_{x}=\frac{1}{S_{y}} \frac{\partial}{\partial y} H_{z}-\frac{1}{S_{z}} \frac{\partial}{\partial z} H_{y}
\end{gathered}
$$

where $S_{y}$ and $S_{z}$ are the stretched coordinate metric coefficients in $y$ and $z$ direction. Initially, these coefficients are chosen via the CFS-PML parameters

$$
s_{v}=\kappa_{v}+\frac{\sigma_{v}}{a_{v}+j \omega \varepsilon_{0}}, v=x, y, z
$$

where $a_{v}, \kappa_{v}$ and $\sigma_{v}$ are assumed to be positive real, and can be one dimensional functions along the $v$-axis.

Transforming (1) or (2) into the time domain leads to a convolution between the inverse of the stretched coordinate parameters and the partial derivatives [8]. Like the dispersive media's manipulation, ADE method can also be used, in which auxiliary variable can be introduced. 1/sy can be expressed as [10]

$$
\frac{1}{s_{y}}=\frac{1}{\kappa_{y}}-\frac{1}{B_{y}}
$$

where

$$
\frac{1}{B_{y}}=\frac{\sigma_{y}}{\kappa_{y}} \frac{1}{\left(\kappa_{y}\left(j \omega \varepsilon_{0}+a_{y}\right)+\sigma_{y}\right)}
$$

By combining (4), the derivative on the right-handed side of (1) can be expressed as

$$
\frac{1}{s_{y}} \frac{\partial}{\partial y} E_{z}=\frac{1}{\kappa_{y}} \frac{\partial}{\partial y} E_{z}+Q_{y, z}^{E}
$$

where the auxiliary variant $Q_{y, z}^{E}$ satisfies

$$
Q_{y, z}^{E}=-\frac{1}{B_{y}} \frac{\partial}{\partial y} E_{z}
$$

Applying (5), (7) can be transformed to time-domain ADE:

$$
\kappa_{y} \varepsilon_{0} \frac{\partial}{\partial t} Q_{y, z}^{E}+\left(\kappa_{y} a_{y}+\sigma_{y}\right) Q_{y, z}^{E}=-\frac{\sigma_{y}}{\kappa_{y}} \frac{\partial}{\partial y} E_{z}
$$

The other components can be manipulated using the same method.

\section{Finite-difference approximation}

The ADE form (8) can be discretized by following finite difference procedure. Without loss of generality, the electric field is sampled on the Yee's cell's edge at integer time step, while the magnetic field is sampled on the center of Yee's cell's face at half integer time step [1]. To be 
compatible with the field's sample, the auxiliary parameter $Q_{y, z}^{E}$ is also sampled at the same position and time with magnetic field. According to this assumption, (8) is discretized as

$$
\kappa_{y} \varepsilon_{0} \frac{Q_{y, z}^{E, n+1 / 2}-Q_{y, z}^{E, n-1 / 2}}{\Delta t}+\left(\kappa_{y} a_{y}+\sigma_{y}\right) \frac{Q_{y, z}^{E, n+1 / 2}+Q_{y, z}^{E, n-1 / 2}}{2}=-\frac{\sigma_{y}}{\kappa_{y}} \frac{\partial}{\partial y} E_{z}^{n}
$$

where the average approximation is used. The explicit expression for updating $Q_{y, z}^{E, n+1 / 2}$ can be achieved from (9)

$$
Q_{y, z}^{E, n+1 / 2}=b_{y} Q_{y, z}^{E, n-1 / 2}-c_{y} \frac{\partial}{\partial y} E_{z}^{n}
$$

where

$$
\begin{gathered}
b_{y}=\frac{2 \kappa_{y} \varepsilon_{0}-\Delta t\left(\kappa_{y} a_{y}+\sigma_{y}\right)}{2 \kappa_{y} \varepsilon_{0}+\Delta t\left(\kappa_{y} a_{y}+\sigma_{y}\right)} \\
c_{y}=\frac{2 \Delta t \sigma_{y}}{\kappa_{y}\left(2 \kappa_{y} \varepsilon_{0}+\Delta t\left(\kappa_{y} a_{y}+\sigma_{y}\right)\right)} .
\end{gathered}
$$

The discretization of (8) is different from the original work by Gedney [10], who sampled the $Q_{y, z}^{E}$ at the integer time step and second term in (8) is not averaged by the neighbor points. He claimed that if the term is averaged, this will lead to instability in the combined ADE-FDTD formulation. However, the numerical experiments in this paper show that the average approach comes out convergent results.

We can see from (11) and (12) that in free space, $b_{y}=1, c_{y}=0$ and $Q_{y, z}^{E}$ sustains zero for all time steps. That is, the expression of ADE-PML becomes field updating equation in free space, just by setting $\sigma=0$. However, the expression of RC-PML cannot be directed deteriorate to free space form and a limit manipulation must be taken account because the denominator of the coefficient become zero when $\sigma \rightarrow 0$. This is an advantage of ADE-PML.

\section{Numerical experiments}

Numerical experiments are now reported which clearly measure the amount of nonphysical reflection a given PML produces as a pulse propagates through a grid boundary [12]. Figure 1 (a) shows two domains on which the two-dimensional FDTD algorithm is computed simultaneously for the transverse magnetic (TM) case. On the boundary of the test domain $\Omega_{T}$ a test PML is applied. Each point in $\Omega_{T}$ has a corresponding member in the substantially larger domain $\Omega_{B}$. A line source is located at grid position $(50,25)$ in both domains. The source produces outward propagating, cylindrical waves which are spatially coincident in both domains up until time steps when the wave interact with the boundary of $\Omega_{T}$. Any reflection from the boundary of $\Omega_{T}$ makes the solution at points within $\Omega_{T}$ differ from the solution at corresponding points within $\Omega_{B}$. The wave solution at points within $\Omega_{B}$ represents the desired numerical modeling of free-space propagation up until time steps when reflections from its own boundary enter the region of $\Omega_{B}$ corresponding to $\Omega_{T}$. By calculating the difference of the solutions in $\Omega_{B}$ and $\Omega_{T}$ at each point at each time step, a measure of the spurious reflection caused by the boundary of $\Omega_{T}$ is obtained.

The difference at $n$th time step is defined

$$
D(i, j)=E_{z}^{T}(i, j)-E_{z}^{B}(i, j)
$$

for all $(i, j)$ within the test domain, where $E_{z}^{T}$ is the solution within $\Omega_{T}$ and $E_{z}^{B}$ is the solution at points within $\Omega_{B}$. $D(i, j)$ is the local error in the test domain caused by its grid boundary reflections. We also define a global reflected error measure 


$$
E=\sum_{i} \sum_{j} D^{2}(i, j)
$$

for all $(i, j)$ within $\Omega_{T}$, which measures the total reflected error within the test grid at the $n_{\text {th }}$ time step. For convenient, the total error is expressed in decibels

$$
\text { error }_{\mathrm{dB}}=10 \log _{10} E
$$

where $E$ is expressed in (14).

The source used in the numerical experiments is the pulse obtained from [12] and is defined as follows:

$$
E_{z}^{n}(50,25)=\left\{\begin{array}{cc}
\alpha\left(10-15 \cos \omega_{1} \xi+6 \cos \omega_{2} \xi-\cos \omega_{3} \xi\right) & \xi \leq \tau \\
0 & \xi>\tau
\end{array}\right.
$$

where

$$
\begin{aligned}
& \alpha=1 / 320 \\
& \omega_{m}=2 \pi m / \tau, m=1,2,3 \\
& \xi=n \Delta t \\
& \tau=10^{-9}
\end{aligned}
$$

and $\Delta t$ is the time step used in the simulation. In the experiments we maintained $\Delta t=2.5 \times 10^{-11}$ sec and $\delta=2 c \Delta t$, where $c$ is the speed of light in free-space and $\delta$ is the space increment of the finite-difference grid. The time profile of the pulse is shown in Figure 1 (b). This pulse was selected because it has an extremely smooth transition to zero. The pulse has its first five derivatives vanish at $\xi=0, \tau$ and has a good approximation to a smooth compact pulse.

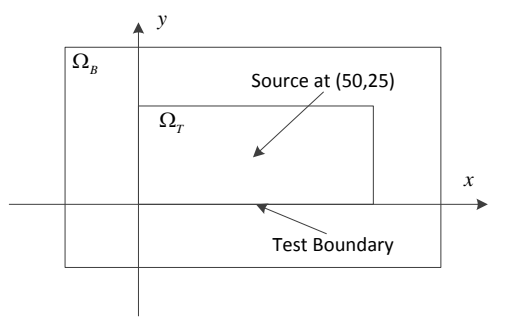

(a) Computational domains

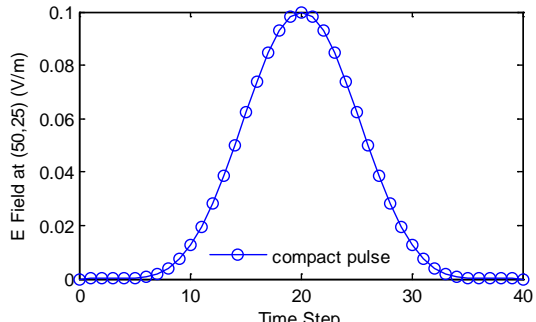

(b) Wave source

Figure 1 Schematic of reflection test calculation domain and incident pulse.

The source point in Figure 1 (a) is 25 cells from the boundary of $\Omega_{T}$ at $y=0$. With the specification $\delta=2 c \Delta t$, disturbances at the source point require 50 time steps to propagate to the boundary at $y=0$. At time step 70, the peak of the pulse just starts to pass through the boundary. We choose to observe the reflection at the first row of grid points away from the $y=0$ boundary at time step $n=100$. This permits the bulk of the outgoing pulse to pass through the boundary and excite the largest observable reflection.

Polynomial scaling is applied to the parameters of PML. $\sigma$ and $\kappa$ are scaled such that they are zero at the interior PML interface and maximum at the terminating boundary. Conversely, $a$ is scaled such that it is a maximum at the FDTD/PML interface and scales to zero at the terminating boundary. This is necessary for the CFS-PML to absorb both evanescent and propagating modes. It has been found that linear scaling for $a$ is optimal [8] and is done so in this paper's numerical experiment.

$\sigma$ is chosen to be spatially variant along the normal axis as

$$
\sigma(z)=\frac{\sigma_{\max }\left|z-z_{0}\right|^{m}}{d_{m}}
$$

where $z_{0}$ is the interface, $d$ is the depth of the PML and $m$ is order of the polynomial variation. This helps to smooth the transition into the PML medium and smaller reflections are encountered. $\sigma_{\max }$ is chosen as 


$$
\sigma_{\max }=\frac{m+1}{150 \pi \delta}
$$

where $\delta$ is spatial increment.

$\kappa$ is chosen as the same spatially variant with $\sigma$, that is

$$
\kappa(z)=\frac{\kappa_{\max }\left|z-z_{0}\right|^{m}}{d_{m}}
$$

In this paper, we set that $\kappa_{\max }=1$ and $a_{\max }=0.05$ and the PML is ten-layer thick.

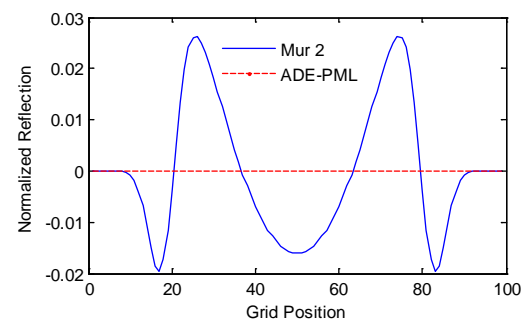

(a) Local error at $n=100$

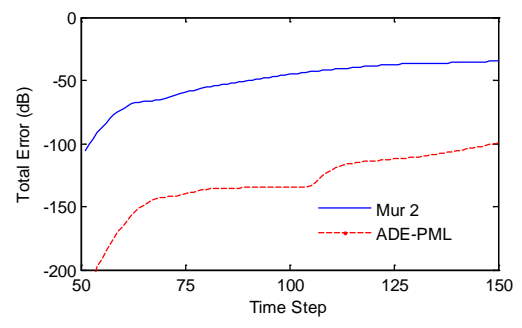

(b) Global error

Figure 2 Reflection error calculation results

Figure 2 shows the local and global reflection errors observed for the ADE-PML. In Figure 2 (a), $D(i, 1)$ has been normalized with the peak value of the incident pulse which strikes the $y=0$ boundary at time step $n=70$ at grid position $(50,0)$. To show the advantage of PML, Mur's second order ABC's absorbing performance is compared. The pulse experiment show that PML do perform much better absorbing effect than Mur's second order ABC.

PML's two implementations: RC and ADE are also compared in Figure 3 with the same constitutive parameters. The results show that the ADE implementation's absorbing performance is better than RC.

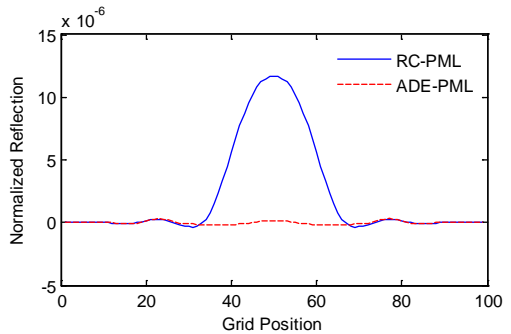

(a) Local error at $n=100$

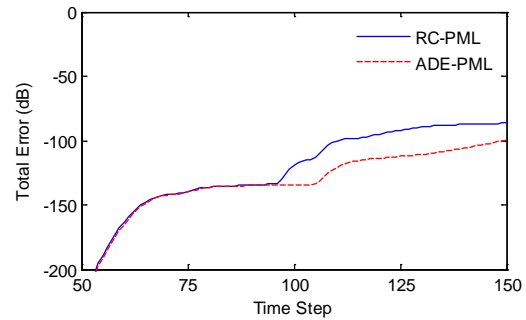

(b) Global error

Figure 3 Error measures, RC and ADE implementations

\section{Conclusion}

In this paper an auxiliary differential form of the complex-frequency shifted PML was implemented based on a stretched-coordinate PML formulation. This is referred to as ADE-PML. Through numerical simulation with the FDTD method, it was validated that the ADE-PML has much better performance than Mur's second order ABC. The RC implementation is also compared and the results show that ADE-PML has better absorbing effect than RC. The expression of ADE-PML can deteriorate to free space form via set $\sigma=0$ and this brings convenient in computer programming.

\section{References}

1.Yee, K.S., Numerical solution of initial boundary value problems involving maxwell's equations in isotropic media. Antennas and Propagation, IEEE Transactions on, 1966. 14(3): p. 302-307.

2.Taflove, A., Application of the Finite-Difference Time-Domain Method to Sinusoidal Steady-State Electromagnetic-Penetration Problems. Electromagnetic Compatibility, IEEE Transactions on, 1980. EMC-22(3): p. 191-202. 
3.Mur, G., Absorbing Boundary Conditions for the Finite-Difference Approximation of the Time-Domain Electromagnetic-Field Equations. Electromagnetic Compatibility, IEEE Transactions on, 1981. EMC-23(4): p. 377-382.

4.Berenger, J.-P., A perfectly matched layer for the absorption of electromagnetic waves. Journal of Computational Physics, 1994. 114(2): p. 185-200.

5.Chew, W.C. and W.H. Weedon, A 3D perfectly matched medium from modified maxwell's equations with stretched coordinates. Microwave and Optical Technology Letters, 1994. 7(13): p. 599-604.

6.Sacks, Z.S., et al., A perfectly matched anisotropic absorber for use as an absorbing boundary condition. Antennas and Propagation, IEEE Transactions on, 1995. 43(12): p. 1460-1463.

7.Gedney, S.D., An anisotropic perfectly matched layer-absorbing medium for the truncation of FDTD lattices. Antennas and Propagation, IEEE Transactions on, 1996. 44(12): p. 1630-1639.

8.Roden, J.A. and S.D. Gedney, Convolution PML (CPML): An efficient FDTD implementation of the CFS-PML for arbitrary media. Microwave and Optical Technology Letters, 2000. 27(5): p. 334-339.

9.Kuzuoglu, M. and R. Mittra, Frequency dependence of the constitutive parameters of causal perfectly matched anisotropic absorbers. Microwave and Guided Wave Letters, IEEE, 1996. 6(12): p. 447-449.

10.Gedney, S.D. and B. Zhao, An Auxiliary Differential Equation Formulation for the Complex-Frequency Shifted PML. Ieee Transactions on Antennas and Propagation, 2010. 58(3): p. 838-847.

11.Luebbers, R., et al., A frequency-dependent finite-difference time-domain formulation for dispersive materials. Electromagnetic Compatibility, IEEE Transactions on, 1990. 32(3): p. 222-227.

12.Moore, T.G., et al., Theory and application of radiation boundary operators. Antennas and Propagation, IEEE Transactions on, 1988. 36(12): p. 1797-1812. 